\title{
Ablation-cooled material removal at high speed with femtosecond pulse bursts
}

\author{
C. Kerse ${ }^{1, *}$, H. Kalaycioglu' ${ }^{2}$, P. Elahi ${ }^{2}$, Ö. Akcaalan ${ }^{2}$, S. Yavaş ${ }^{3}$, M. D. Aşik ${ }^{4}$, D. K. \\ Kesim $^{1}$, K. Yavuz ${ }^{2}$, B. Çetin ${ }^{5}$, and F. Ö. Ilday ${ }^{1,2}$ \\ ${ }^{1}$ Department of Electrical and Electronics Engineering, Bilkent University, Ankara, 06800, Turkey \\ ${ }^{2}$ Department of Physics, Bilkent University, Ankara, 06800, Turkey \\ ${ }^{3}$ FiberLAST, A.Ş., Ankara, 06800, Turkey \\ ${ }^{4}$ Nanotechnology and Nanomedicine Department, Hacettepe University, Ankara, 06800, Turkey \\ ${ }^{5}$ Department of Mechanical Engineering, Bilkent University, Ankara, 06800, Turkey \\ *cankerse@ee.bilkent.edu.tr
}

\begin{abstract}
We report exploitation of ablation cooling, a concept well-known in rocket design, to remove materials, including metals, silicon, hard and soft tissue. Exciting possibilities include ablation using sub-microjoule pulses with efficiencies of $100-\mu \mathrm{J}$ pulses.
\end{abstract}

OCIS codes: $140.3390,060.1510,320.7090$

Femtosecond pulses hold great promise for high-precision material and tissue processing. It is well-known that use of such pulse durations allows very precise and virtually thermal-damage free material removal under appropriate conditions. However, two drawbacks remain. The first is the limited ablation rates, which is particularly limiting for biological tissue removal. The second is that at several microjoules, but often 10's to 100's of microjoules of pulse energy are required. Regarding speed of ablation, the physics of the laser-material interaction precludes a straightforward scaling up of the removal rate simply by sending pulses more frequently, since this leads to collateral damage due to heat accumulation.

Here, we exploit the same physics to circumvent this limitation. We apply rapid succession of ultrafast pulses from a burst-mode fibre laser to ablate the target before residual heat deposited by the previous pulse can diffuse away from the interaction region. In addition to being able to process thermally sensitive materials, such as brain tissue, at high speed and without thermal damage, our approach reduces the required pulse energy by orders of magnitude, opening the route to highly efficient material removal with oscillator-level pulse energies.

For a long time, it was commonly assumed that heat effects are nearly completely eliminated through the use of ultrafast pulses. Heat damage can indeed occur during ultrafast pulse processing as a result of pulse-to-pulse accumulation of residual heat that is deposited around the border of the ablated region by each pulse. While deposition of some residual heat by each pulse is unavoidable, we present here a laser system that can catch much of this heat before it can diffuse beyond the volume to be ablated by the next incoming pulse by operating at very high repetition rates. This brings three interrelated advantages: (1) Most of the residual heat left by the previous pulse has not yet diffused out of the volume to be ablated by the next pulse. Thus, each pulse targets an already hot volume, which lowers the required ablation energy and peak power with numerous side benefits, such minimizing plasma shielding, reducing shock wave, cavitation bubble formation and self-focusing. In addition, the quantity of residual heat is proportional to the pulse energy, thus reducing the magnitude of the problem to be solved. (3) Finally and most importantly, much of this residual heat is then carried away from the tissue in the form of ablated matter when ablated by the next pulse, reducing the build-up of heat from pulse to pulse. This is known as ablation cooling, which is very well known in the context of atmospheric entry of meteorites or reusable space rockets and has been routinely used in rocket design since 1950's. In the case of laser ablation of tissue, ablation is not continuous, but occurs only during and shortly after the pulses. In order to benefit from ablation cooling, the repetition rate must exceed a critical value, which scales with the thermal conductivity of the target material, and, in practice, at least tens of $\mathrm{MHz}$.

Given that the minimum pulse energy is substantial, continuous delivery of such repetition rates corresponds to prohibitively high average powers (which can amount to hundreds of watts). To overcome this bottleneck, we have implemented burst-mode operation, which was first demonstrated by R. Marjoribanks, et al. using picosecond solid state lasers [1]. In burst mode, the laser produces packages of high-repetition-rate pulses, or bursts, which are repeated at a much lower, adjustable frequency. In this work, we utilized fiber lasers developed in our laboratory [2].

We demonstrate that the maximum levels of ablation per pulse energy reported using high pulse energies can be attained with much lower pulse energies with optimized burst parameters. After demonstrating this effect on silicon 
and copper (Fig. 1), we applied this approach to processing of various targets of medical interest, namely, dentine (as example of hard tissue), agar (representative of opthalmic tissue), cow eyes and mouse brain tissue (Fig 2.). In all of these experiments, we obtained excellent results with the burst mode. Specifically, we report thermal-damage-free ablation of brain tissue at $0.75 \mathrm{~mm}^{3} / \mathrm{min}$, representing an order of magnitude improvement on previous results, along with a potential to increase ablation speed by two orders of magnitude. These results pave the way towards in vivo operation at medically relevant speeds, eventually delivered via flexible fibres to hard-to-reach targets.

(a)

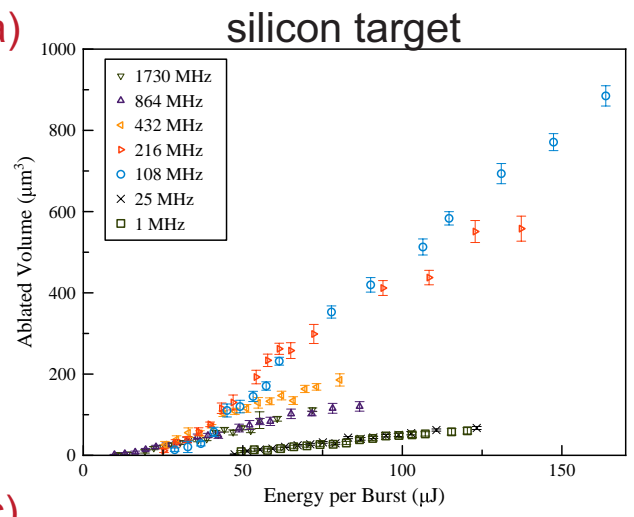

(c)

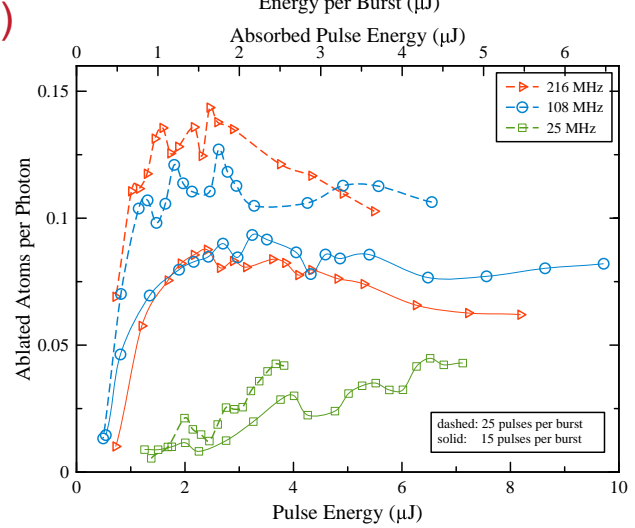

(b)

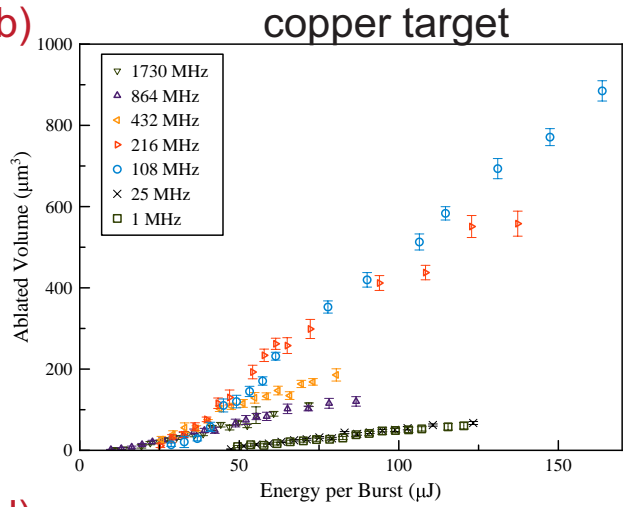

(d)

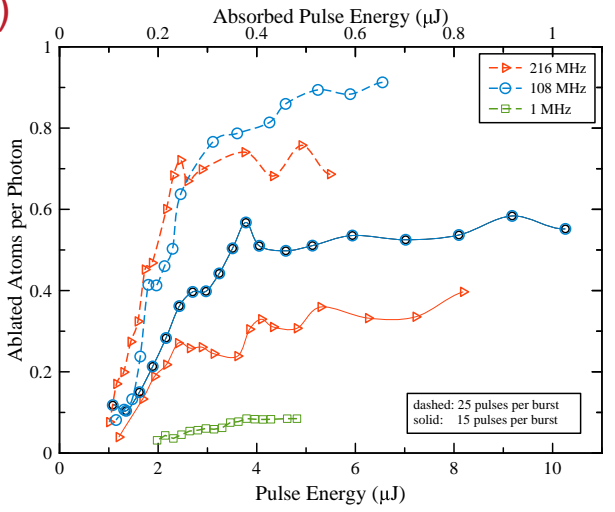

Fig. 1. Ablation results obtained at various intra-burst repetition rates on silicon and copper targets. Ablated volume by a single pulse burst ( 25 pulses within the burst) at various intra-burst repetition rates for (a) silicon and (b) copper targets. Ablation efficiency (number of atoms per incident photon) for select repetition rates for (c) silicon and (d) copper targets.

Careful analysis of the ablation results on silicon and copper targets reveals several important features. First, in every case tested, burst-mode operation is much more efficient than uniform-mode operation of the laser (Fig. 1a and $1 \mathrm{~b}$ ). The total ablated volumes, e.g., for copper per single pulse burst meet and even exceed the values reported previously using uniform pulsed lasers at much higher fluence levels [3]. In other words, 25 individual pulses making up a 150 microjoule burst ablate roughly as much material as a single 150 microjoule pulse. This changes completely the requirements on laser technology for efficient ultrafast material processing.

Second, we inspect the ablation efficiencies, shown in Fig. 1c and 1d in units of atoms ablated per laser photon. In each case, increasing number of pulses within a burst increases the efficiency. More importantly, going from 108 $\mathrm{MHz}$ to $216 \mathrm{MHz}$, the efficiency difference is less for 25 pulse/burst than for 15 pulses/burst for a copper target. For a silicon target, $216 \mathrm{MHz}$ is even more efficient until the highest energies. At any rate, differences in efficiency are very small. These data suggest strongly that roughly the same efficiencies can be maintained if intra-burst repetition rate is increased, while increasing simultaneously the number of pulses within the burst, and decreasing energy per pulse (keeping the burst energy constant). Currently, experiments are under way in our laboratory to test this expectation. If verified, the implication is that hundreds of sub-microjoule individuals pulses within a single burst can be about as efficient as a 100-microjoule single pulse.

In conclusion, we report exciting results on processing of a large variety of materials, including various metals 
(however, only copper is shown in Fig. 1), silicon, agar, dentine, brain and eye tissue. In all cases, burst-mode operation excels, for which we provide a simple physical explanation. Results on non-biological targets are exciting in their own right, required individual pulse energies can be decreased by orders of magnitude, which challenges the accepted paradigm in terms of required pulse parameters. This, in turn, has major implications for the required laser technology. However, we are even more excited by results on biological targets, where we have achieved ablation rates of 0.75 $\mathrm{mm}^{3} / \mathrm{min}$ for brain tissue. These results pave the way towards in vivo operation at medically relevant speeds, eventually delivered via flexible fibers to hard-to-reach targets.

(a)

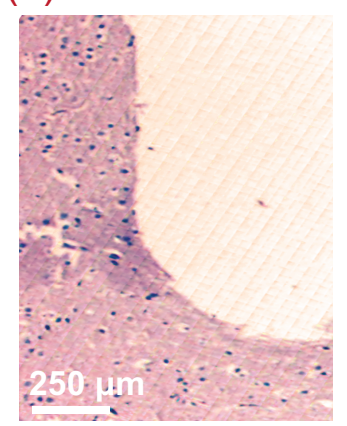

(e)
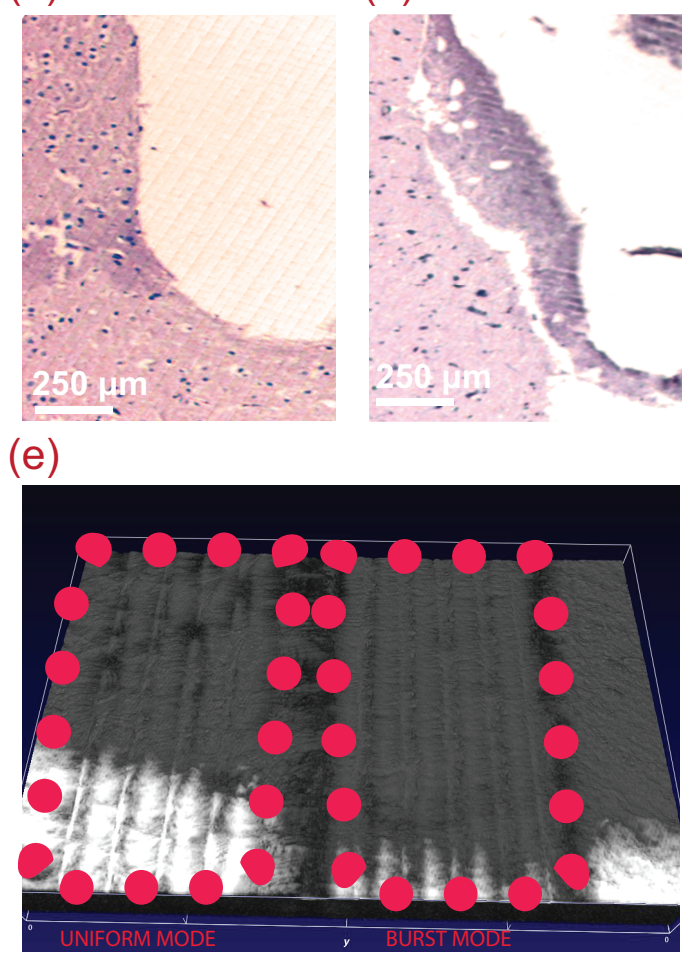

(b) (c)

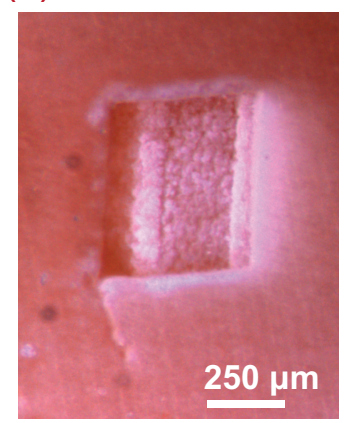

(f)

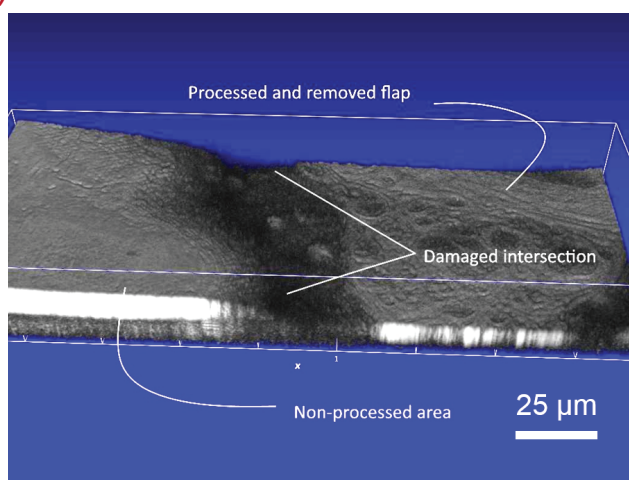

Fig. 2. Burst-mode and uniform mode results obtained for various hard and soft tissue targets. We note that in all the comparisons, all lasers parameters are kept identical apart from the sequencing of the pulses (uniform or burst mode) to ensure fair comparison. Histological analysis of brain tissue processed using (a) burst-mode and (b) uniform-mode operation of the laser, showing burstmode ablation is free of thermal damage seen with uniform mode. Results of processing of dentine using (c) burst-mode and (d) uniform-mode operation. Processing of agar using (e) burst-mode and uniform-mode operation, showing cleaner and deeper cuts with burst mode. Results of a preliminary flap removal operation using burst-mode on cow eye.

\section{References}

1. M. Lapczyna, K. P. Chen, P. R. Herman, H. W. Tan, and R. S. Marjoribanks, "Ultra high repetition rate (133 MHz) laser ablation of aluminum with 1.2-ps pulses," Appl. Phys. A 69, 883886 (1999).

2. H. Kalaycoglu, O. Akcaalan, S. Yavas, Y. B. Eldeniz, and F. O. Ilday, "Burst-mode Yb-doped fiber amplifier system optimized for low-repetition-rate operation,” J. Opt. Soc. Am. B 32, 900-906 (2015).

3. H. Furukawa, M. Hashida, "Simulation on femto-second laser ablation," Appl. Surf. Sci. 197, 114-117 (2002). 